\title{
Relaxation of a nonequilibrium phonon distribution induced by femtosecond laser irradiation
}

\author{
Isabel Klett* and Bärbel Rethfeld \\ Department of Physics and OPTIMAS Research Center, Technical University of Kaiserslautern, \\ Erwin Schroedinger Str. 46, 67663 Kaiserslautern, Germany
}

(Dated: October 2, 2018)

\begin{abstract}
Ultrafast laser irradiation of solids leads to a thermodynamic nonequilibrium within and between the electron and phonon subsystems of the material. Due to electron-electron and phonon-phonon collisions, both subsystems relax into respective new thermodynamic equilibria within a characteristic thermalization time, which is different for each one of them. Moreover, they equilibrate their temperatures by electron phonon coupling. The relaxation of the electronic nonequilibrium and its effect on the electron phonon coupling was subject to a number of studies and it is comparably well understood, while the nonequilibrium within the phononic subsystem is usually neglected and its influence of the nonequilibrium phonons on other relaxation processes is unclear. Our calculations show significant differences in the energy transfer rate between the electrons and the phonons depending whether a nonequilibrium distribution is assumed for the phonons or not. Here, we present a model to study the relaxation of the nonequilibrium phonon subsystem. Collisions between phonons are described within the frame of Boltzmann integrals. From this, an energy-dependent relaxation time can be extracted and inserted into a relaxation-time approach. Within the frame of this model, we study the thermalization of a phonon distribution induced by ultrafast laser irradiation. We show, that the thermalization time of such a distribution is of the order of some hundreds of picoseconds. Moreover, we discuss the energy transfer between Fermi-distributed electrons and nonequilibrium phonons and compare this to the energy transfer for equilibrium distributions in both subsystems.
\end{abstract}

\section{INTRODUCTION}

In the past decades, the possibility to generate very short laser pulses of high intensity has emerged. With simple mode-locked laser systems, pulse durations below one picosecond can be achieved. By applying more advanced laser systems, even the generation of laser pulses in the attosecond regime has been reported $[1,2]$. There is a wide range of applications for ultrashort laser pulses in research, industry and medicine [3,4]. The option to generate ultrashort laser pulses opens up the opportunity to study microscopic processes in solids on an ultrashort time scale. Such processes have been a subject to numerous studies in the past 20 years [5-15], specifically for phonons reaching from experimental measurements of coherent phonons [16-18], to DFT calculations that predict ultrafast phonon bandstructure changes after laser excitation [19-21].

The irradiation of a solid with a femtosecond laser pulse leads to a nonequilibrium state within the electron system as well as between the electron and phonon subsystems, due to the fact that the laser energy is mainly absorbed by the electrons, while the lattice remains nearly unaffected. Following the excitation, two main processes occur: (1) The electrons thermalize due to electron-electron collisions, a process that takes place on a timescale of a few up to several hundreds of femtoseconds $[12,15,22,23]$. (2) The electrons transfer the absorbed laser energy to the lattice due to the electronphonon coupling. The lattice temperature is rising, while the electrons are cooled down, until a new thermodynamic equilibrium between the electron and phonon systems is reached. This process usually takes place on a timescale of a few picoseconds [23].

A commonly applied minimal model that describes the energy relaxation of electrons and phonons after laser irradiation is the well-known two-temperature model (TTM) [24]. Within this model, the electrons and phonons are described with different temperatures after laser irradiation, because only the electrons are initially heated by the laser. The TTM is widely applied to describe the energy relaxation between electrons and phonons [25-27]. Since this model is based on temperatures, it implicitly assumes equilibrium states within each of both subsystems. Therefore, the application of the TTM on a femtosecond timescale after laser irradiation is questionable. The limits of the two-temperature model in the description of the relaxation dynamics of electrons and phonons after laser irradiation have been addressed in several studies $[8,15,23,28-31]$. However, in most of the studies considering the electron relaxation $[8,13,15,32,33]$ the phonon system is assumed to be thermalized. Only recently, the thermalization of the phonon system has been studied by solving a simplified Boltzmann integral [34].

In [35] it was shown, that the electrons do not couple to all phonon modes equally. For example, in metals, preferably the longitudinal mode absorbs the electron energy, so the assumption of thermalized phonons after femtosecond laser irradiation does not hold. The phonons don't absorb the energy of the electrons equally, so, after the exciation of a solid with a femtosecond laser pulse, the phonon subsystem will be in a nonequilibrium state. The phonons thermalize due to phonon-phonon collisions, a process, which takes place on a timescale of several picoseconds [13]. The impact of a phonon nonequilibrium 
on the electron-phonon coupling and the electron distribution is unclear.

In the present work, we study the thermalization of a model nonequilibrium phonon distribution by solving Boltzmann collision integrals, from which we can extract phonon relaxation times. These are inserted into a relaxation time approach, which gives us insights on the temporal evolution of the phonon distribution. Furthermore, we study the influence of the nonequilibrium phonons on the energy transfer between the electron and phonon systems of the material.

The paper is organized as follows: First, a model nonequilibrium distribution for phonons after laser irradiation is introduced. Then, we derive the Boltzmann collision integral for the description of phonon-phonon processes and apply it to the model nonequilibrium distribution. From the calculated collision term, we extract wavenumber-dependent relaxation times and insert them into a relaxation time approach. This gives an idea of the temporal evolution of this nonequilibrium phonon distribution. In the last part of the paper, we check the influence of nonequilibrium phonons on the electronic system by calculating energy transfer rates between Fermidistributed electrons and phonons in nonequilibrium and compare them to energy transfer rates with both subsystems in equilibrium.

\section{KINETIC DESCRIPTION}

In this work we describe the change of the distribution functions of electrons and phonons within the frame of the Boltzmann equation. Although it originates in the kinetic theory of gases, the Boltzmann equation was already successfully applied in the description of quasiparticles in solids [8,11-13]. We do not consider transport effects and external force fields. Considering collisions between electrons and phonons and collisions within the respective subsystems, as well as the laser excitation, we end up with the following form of the Boltzmann equation for electrons and phonons

$$
\begin{gathered}
\frac{\partial f}{\partial t}=\Gamma_{e l-e l}+\Gamma_{e l-p h}+\Gamma_{a b s}^{*} \\
\frac{\partial g}{\partial t}=\Gamma_{p h-p h}+\Gamma_{p h-e l}+\Gamma_{a b s}^{*} .
\end{gathered}
$$

The terms $\Gamma_{i}$ hereby represent complete collision integrals. $\Gamma_{e l-p h}$ and $\Gamma_{p h-e l}$ describe the collisions between electrons and phonons due to the electron-phonon coupling, $\Gamma_{e l-e l}$ denotes the change of the electron distribution function due to electron-electron collisions, which are responsible for the thermalization of the electronic subsystem. In analogy, the phonon-phonon collisions are represented by $\Gamma_{p h-p h}$. This collision term describes the thermalization of the phonon system of the material. Finally, $\Gamma_{a b s}^{*}$ stands for the absorption of the laser energy, which enters the electronic system, in case that an optical laser pulse was applied. If the frequency of the laser pulse was in the terahertz regime, it would be possible to exciten the phonons directly [36]. Most of these collison integrals are well known and have already been applied in the description of the temporal evolution of the electron and phonon system after femtosecond laser irradiation $[8,11,13,15]$. The phonon-phonon collision term so far was only calculated with severe simplifications [34] in the phonon-phonon matrix element. This study will concentrate on this phonon-phonon collision term, which is derived below.

We apply our collision term to an assumed nonequilibrium distribution of the phonons after laser excitation. Such distributions were calculated for instance in Ref [11].

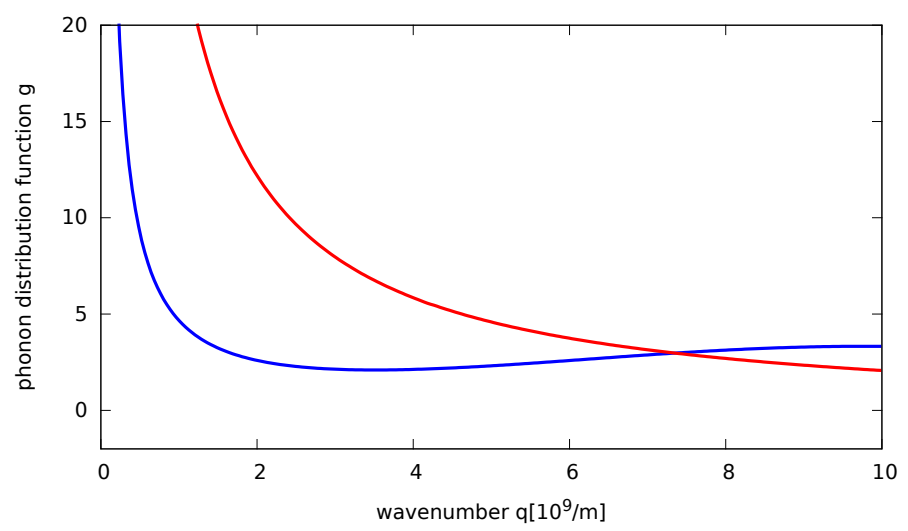

FIG. 1: Model of an examplary nonequilibrium phonon distribution after excitation by hot electrons (blue) and

a Bose distribution of equal internal energy (red).

Figure 1 shows the applied nonequilibrium distribution and a Bose distribution

$$
g(q, T)=\frac{1}{e^{\frac{\hbar q c_{s}}{k_{B} T}}-1} .
$$

of the same internal energy. For small wavenumbers $q$, the distribution resembles a Bose distribution of lower internal energy than the nonequilibrium distribution, for larger wavenumbers, the occupation number is higher. A similar trend of the nonequilibrium distribution for phonons after laser irradiation was calculated in Ref [11]. The internal energy of the Bose distribution corresponds to a temperature of 1634 Kelvin, that is slightly lower than the melting temperature of silicon.

\section{PHONON-PHONON COLLISIONS}

This study deals with the relaxation of nonequilibrium phonons. In the following, we present the derivation of the phonon-phonon collision term $\Gamma_{p h-p h}$.

For many studies, isotropic collision terms can be assumed $[13,15]$. However, this assumption is insufficient 

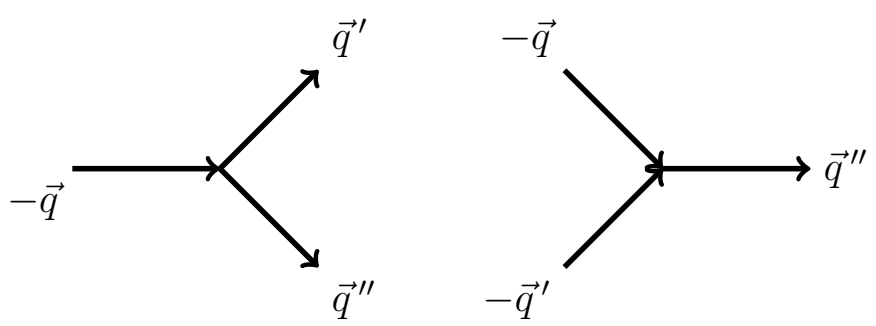

FIG. 2: Possible interaction processes between three phonons. Either one phonon decays into two others or two phonons combine into one.

for the description of phonon-phonon collisions. The phonon-phonon collision term has to be kept directiondependent, in order to distinguish between the different phonon modes. Phonon-specific quantities like frequencies and polarization vectors follow from the chosen interatomic potential and the crystal structure of the material. These quantities are material specific and enter the matrix element for phonon-phonon collisions.

The most simple interaction processes between phonons are the creation or annihilation of one phonon. As shown in figure 2, both of these processes contain three particles. In the creation process one phonon decays into two other phonons, in the annihilation process two phonons combine into one. $\vec{q}$ hereby denote the wavevectors of the participating phonons, $\vec{q}^{\prime}$ and $\vec{q}^{\prime \prime}$ stand for the collision partners. To describe these processes, the harmonic approximation for the Hamiltonian is not sufficient, instead a Taylor expansion of the effective potential up to the third order is required. Therefore, the chosen interatomic potential has to be at least a three-body potential. In our case, we apply a Stillinger-Weber potential [37]. The Hamiltonian for phonon-phonon interactions denotes as [35]

$$
\begin{aligned}
H_{p h-p h} & =\frac{1}{3 !} \sum_{\vec{\ell}, \vec{b}, \vec{\ell}^{\prime}, \vec{b}^{\prime}, \vec{\ell}^{\prime \prime}, \vec{b}^{\prime \prime}} \sum_{\alpha, \beta, \gamma} r_{\vec{\ell} \vec{b}}^{\alpha} r_{\vec{\ell}^{\prime} \vec{b}^{\prime}}^{\beta} r_{\vec{\ell}^{\prime \prime} \vec{b}^{\prime \prime}}^{\gamma} \\
& \cdot\left(\frac{\partial^{3} V}{\partial \vec{r}_{\overrightarrow{\ell b}} \partial \vec{r}_{\vec{\ell}^{\prime} \vec{b}^{\prime}} \partial \vec{r}_{\vec{\ell}^{\prime \prime}} \vec{b}^{\prime \prime}}\right)_{\alpha \beta \gamma},
\end{aligned}
$$

where the $\vec{r}_{\overrightarrow{l b}}$ represent the displacement vectors of the atoms out of their equilibrium position. The vectors $\vec{\ell}$ hereby denote vectors to the origins of the unit cells, $\vec{b}$ the vectors from the origin of the unit cell to the different atoms. The primed quantities refer to the respective displaced atoms. The term contains a triple inner product between the displacement vectors and the tensor of the third derivative with respect to the interatomic potential to the displacement vectors written in component notation as sum over $\alpha, \beta, \gamma$. Following some transformations [35], the phonon-phonon interaction Hamiltonian can be rewritten as a term consisting of creation and annihila- tion operators

$$
\begin{array}{r}
H_{p h-p h}=\frac{1}{3 !} \sum_{\vec{q}, p, \vec{q}^{\prime}, p^{\prime}, \vec{q}^{\prime \prime}, p^{\prime \prime}} \delta_{\vec{G}, \vec{q}+\vec{q}^{\prime}+\vec{q}^{\prime \prime}} F_{\alpha \beta \gamma}\left(a_{\vec{q} p}^{+}-a_{-\vec{q} p}\right) \\
\cdot\left(a_{\vec{q}^{\prime} p^{\prime}}^{+}-a_{-\vec{q}^{\prime} p^{\prime}}\right)\left(a_{\vec{q}^{\prime \prime} p^{\prime \prime}}^{+}-a_{-\vec{q}^{\prime \prime} p^{\prime \prime}}\right) \mathcal{F}_{\vec{q} p \vec{q}^{\prime} p^{\prime} \vec{q}^{\prime \prime} p^{\prime \prime}}
\end{array}
$$

with $\vec{G}$ denoting a reciprocal lattice vector, $p$ denoting the phonon modes, the primed quantities hereby denote the modes of the collision partners, and

$$
\begin{aligned}
\mathcal{F}_{\vec{q} p \vec{q}^{\prime} p^{\prime} \vec{q}^{\prime \prime} p^{\prime \prime}}= & i\left(\frac{1}{2} \hbar\right)^{3 / 2}\left(N V_{K}\right)^{-1 / 2}\left(\nu_{\vec{q} p} \nu_{\vec{q}^{\prime} p^{\prime}} \nu_{\vec{q}^{\prime \prime} p^{\prime \prime}}\right)^{-1 / 2} \\
& \cdot \sum_{\vec{b}, \vec{b}^{\prime}, \vec{b}^{\prime \prime}}\left(m_{\vec{b}} m_{\vec{b}^{\prime}} m_{\vec{b}^{\prime \prime}}\right)^{-1 / 2} \sum_{\alpha, \beta, \gamma} \mathbf{e}_{\vec{q} \vec{b} p}^{\alpha} \mathbf{e}_{\vec{q}^{\prime} \vec{b}^{\prime} p^{\prime}}^{\beta} \mathbf{e}_{\vec{q}^{\prime \prime} \vec{b}^{\prime \prime} p^{\prime \prime}}^{\gamma} \\
& \cdot \sum_{\vec{h}^{\prime}, \vec{h}^{\prime \prime}} e^{-i \vec{q}^{\prime} \vec{h}^{\prime}} e^{-i \vec{q}^{\prime \prime} \vec{h}^{\prime \prime}}\left(\frac{\partial^{3} V}{\partial \vec{r}_{\overrightarrow{\ell b}} \partial \vec{r}_{\vec{\ell}+\vec{h}^{\prime}, \vec{b}^{\prime}} \partial \vec{r}_{\vec{\ell}+\vec{h}^{\prime \prime}, \vec{b}^{\prime \prime}}}\right)_{\alpha \beta \gamma} .
\end{aligned}
$$

This Hamiltonian contains the triple inner product of the polarization vectors $\overrightarrow{\mathbf{e}}$ with the tensor of the third derivatives of the interatomic potential with respect to the atom displacement vectors. Additionally, we define the vectors $\vec{h}^{\prime}$ and $\vec{h}^{\prime \prime}$ as $\overrightarrow{\ell^{\prime}}=\vec{\ell}+\vec{h}^{\prime}$ and $\vec{\ell}^{\prime \prime}=\vec{\ell}+\vec{h}^{\prime \prime}$. The $\nu$ denote the phonon frequencies, the $m$ are the atomic masses and $N V_{K}$ is the product of the considered crystal volume and the number of unit cells per volume. The phonon frequencies and polarisation vectors were calculated from the eigenvalues and eigenvectors of the dynamical matrix.

\section{A. The Phonon-Phonon collision term}

With the Hamiltonian for three phonon interactions derived above, Fermi's golden rule can be applied to derive a Boltzmann collision term for phonon-phonon scattering processes leading to a collision term

$$
\Gamma_{p h-p h}=\frac{2 \pi}{\hbar}\left|\left\langle 1\left|H_{p h-p h}\right| 0\right\rangle\right|^{2} \delta\left(E_{1}-E_{0}\right) .
$$

To evaluate this expression, the commutation relations valid for phonons

$$
\left[a_{i}, a_{j}^{+}\right]=\delta_{i j},\left[a_{i}^{+}, a_{j}^{+}\right]=0
$$

are applied. Additionally, the following terms for creation and annihilation operators apply in the case of phonons

$$
<a_{i}^{+} a_{i}>=g\left(E_{i}\right)
$$

and

$$
<a_{i} a_{i}^{+}>=\left(1+g\left(E_{i}\right)\right)
$$


with the phonon distribution function $g$ at given energy $E_{i}$, with $E=\hbar c_{s} q$. Taking all these relations into account, a Boltzmann collision term for phonon-phonon interactions is calculated

$\Gamma_{\mathrm{ph}-\mathrm{ph}}(\vec{q})=\frac{2 \pi}{\hbar}\left(\frac{1}{3 !}\right)^{2} \sum_{p^{\prime}, p^{\prime \prime}} \sum_{\vec{q}^{\prime}, \vec{q}^{\prime \prime}} \delta_{\vec{G}, \vec{q}+\vec{q}^{\prime}+\vec{q}^{\prime \prime}}|M|^{2} \mathcal{G} \delta_{E_{1}, E_{0}}$

with

$$
\begin{aligned}
M & =i\left(\frac{1}{2} \hbar\right)^{3 / 2}\left(N V_{K}\right)^{-1 / 2} \sum_{b, b^{\prime}, b^{\prime \prime}}\left(m_{\vec{b}} m_{\vec{b}^{\prime}} m_{\vec{b}^{\prime \prime}}\right)^{-1 / 2} \\
& \cdot\left(\nu_{\vec{q} p} \nu_{\vec{q}^{\prime} p^{\prime}} \nu_{\vec{q}^{\prime \prime} p^{\prime \prime}}\right)^{-1 / 2} \sum_{\alpha, \beta, \gamma} \mathbf{e}_{\vec{q} \vec{b} p}^{\alpha} \mathbf{e}_{\vec{q}^{\prime} \vec{b}^{\prime} p^{\prime}}^{\beta} \mathbf{e}_{\vec{q}^{\prime \prime} \vec{b}^{\prime \prime} p^{\prime \prime}}^{\gamma} \\
& \cdot \sum_{\vec{h}, \vec{h}^{\prime}} e^{-i \vec{q}^{\prime} \vec{h}^{\prime}} e^{-i \vec{q}^{\prime \prime} \vec{h}^{\prime \prime}}\left(\frac{\partial^{3} V}{\partial r_{\ell b} \partial r_{\vec{\ell}+\vec{h}^{\prime}, \vec{b}^{\prime}} \partial r_{\vec{\ell}+\vec{h}^{\prime \prime}, \vec{b}^{\prime \prime}}}\right)_{\alpha \beta \gamma},
\end{aligned}
$$

and

$$
\begin{gathered}
\mathcal{G}=(g+1) g_{-}^{\prime}\left(g^{\prime \prime}+1\right)-g_{-}\left(g^{\prime}+1\right)\left(g^{\prime \prime}+1\right)-g_{-} g_{-}^{\prime}\left(g^{\prime \prime}+1\right) \\
+(g+1)\left(g^{\prime}+1\right) g_{-}^{\prime \prime}+(g+1) g_{-}^{\prime} g_{-}^{\prime \prime}-g_{-}\left(g^{\prime}+1\right) g_{-}^{\prime \prime}
\end{gathered} .
$$

The functional $\mathcal{G}$ represents a term over the distribution functions of the phonons, indicating the gain and loss of phonons in the different states. The abbreviation $g$ here refers to the value of the distribution $g(E(\vec{q}))$ at the given state.

The phonon-phonon collision term contains multiple sums, two are sums over the wave vectors $\vec{q}$ of the scattered phonons, the others run over the modes $p$, respectively. The sums over the wave vectors can be rewritten in integral form leading to six integrals over the components of the wave vectors. By means of an evaluation of the delta functions representing energy and momentum conservation, the number of integrals required can be reduced to two.

In figure 3, the temporal derivative of the assumed nonequilibrium phonon distribution (see Figure 1) is shown.

For the calculation we took a material with an fcc structure, so only acoustic phonons are possible. We applied material parameters of silicon, the excitend phonon mode is the longitudinal one. We apply a Debye temperature of 645 Kelvin, a sound velocity of $8433 \frac{\mathrm{m}}{\mathrm{s}}$ an atomic mass of $28.0855 \mathrm{u}$ and a lattice parameter of $5.431 \mathrm{~A}$. We consider atomic displacements and phonon wavevectors in (111) direction.

\section{B. Temporal evolution of the phonon distribution}

Calculating the complete temporal evolution of the phonon system with the Boltzmann collision integral

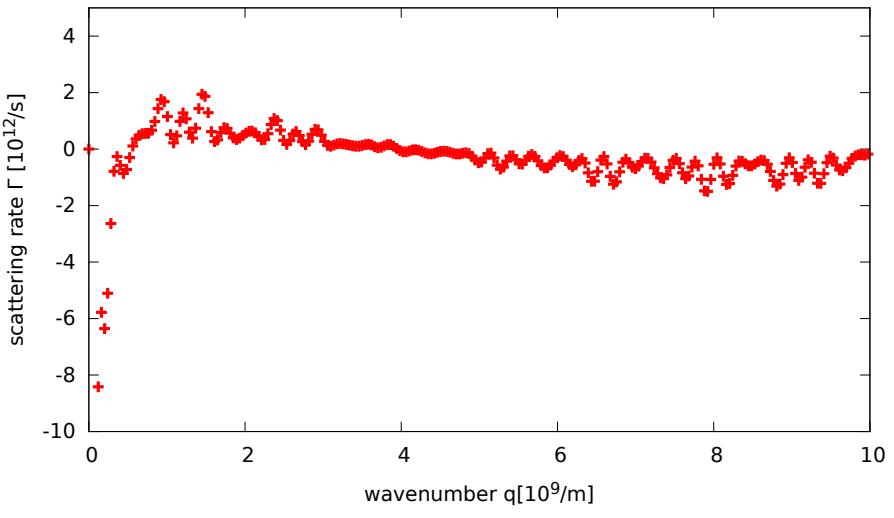

FIG. 3: Collision term $\Gamma_{p h-p h}$ according to Equation (11) of the assumed nonequilibrium phonon distribution function, shown in Figure 1 .

would be extremely time-consuming. A compromise would be a relaxation time approach. The assumption hereby is, that a system evolves towards an equilibrium state due to collision processes. Within this approximation, the time derivative of the distribution function writes as following

$$
\Gamma_{p h-p h}=\frac{\partial g(q)}{\partial t}=\frac{g(q)-g_{\text {Bose }}(q)}{\tau(q)} .
$$

The change of the nonequilibrium distribution $g$ is thus given by its difference to a Bose distribution of equal internal energy divided by the relaxation time $\tau$. All quantities depend on the phonon wave number $q$, and therefore also on the phonon energy.

Since we determine the time derivative of the phonon distribution $g(q)$ through the Boltzmann collision integral, we can extract relaxation times, which depend on the wavenumber $q$

$$
\tau(q)=\left|\frac{g(q)-g_{\text {Bose }}(q)}{\Gamma_{p h-p h}}\right| .
$$

A problem of this approach is that the internal energy is not exactly conserved when applying wave-numberdependent relaxation times. Therefore, we checked in the following calculations, that these deviations are relatively small. The highest deviation in the internal energy is found to be around $9 \%$.

The relaxation times of the nonequilibrium phonon distribution shown in figure 1 after laser irradiation were calculated through equation (15) and are shown in figure 4. The relaxation times for small wave numbers are on a timescale of up to some hundreds of picoseconds, while for higher wavenumbers, the relaxation times are much smaller. These results indicate that the phonon distribution thermalizes on timescales of a few hundred picoseconds.

The wavenumber-dependent relaxation times can be inserted into a relaxation time approach (15) and integrated in time. This gives an idea of the temporal evolution of the phonon distribution. 


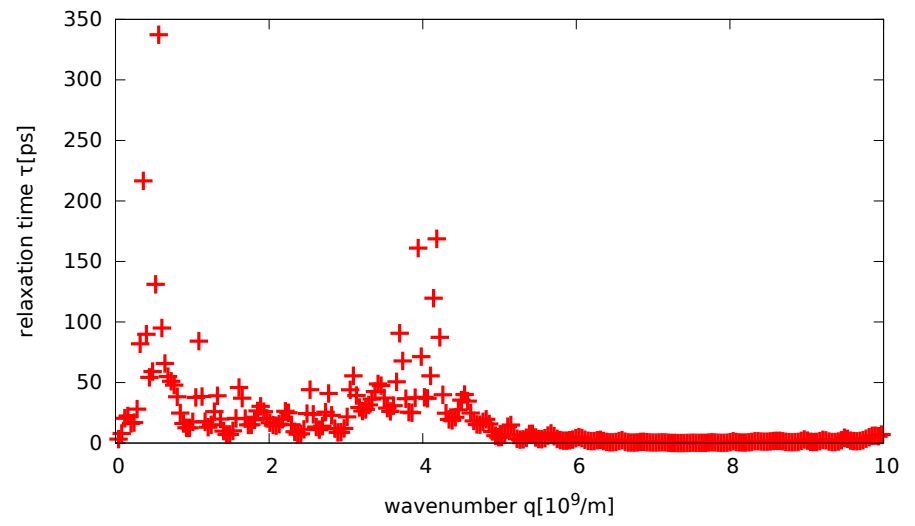

FIG. 4: Calculated phonon relaxation times depending on the wavenumber $q$.

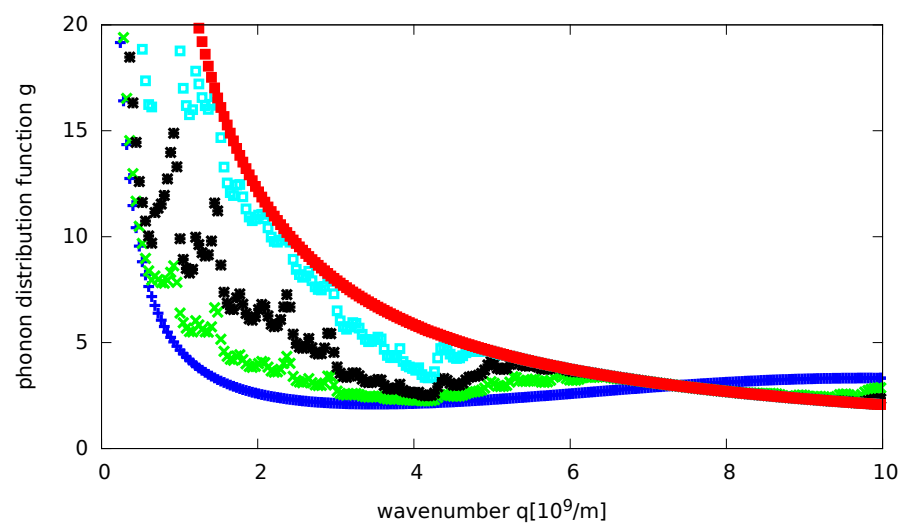

FIG. 5: Relaxation of a phonon nonequilibrium distribution. The plotted timesteps are the initial nonequilibrium distribution (blue), the distribution after 3 ps (green), 10 ps (black), 40 ps (light blue) and the relaxed distribution after 500 ps (red).

In figure 5, such temporal evolution of the nonequilibrium distribution function within $500 \mathrm{ps}$ is plotted. A comparatively fast relaxation of the nonequilibrium distribution at higher wavenumbers can be observed, where it is already almost thermalized after 3 picoseconds, while the thermalization of the whole distribution function to a new equilibrium distribution takes much longer. After 40 picoseconds, the distribution still deviates from an equilibrium distribution, particularly at small wave numbers. This indicates that processes driven by phonons of small energy are influenced by the phononic nonequilibrium on timescales in the 100 ps range.

\section{INFLUENCE OF A PHONON NONEQUILIBRIUM ON THE ELECTRON-PHONON ENERGY TRANSFER}

It is interesting to check how strong the influence of a nonequilibrium distribution of the phonons on the electron system and on the coupling between electrons and phonons is. It was already shown, that nonequilibrium electrons influence the electron phonon coupling $[8,15,23,38]$. Therefore, it can be expected, that nonequilibrium phonons also affect the energy relaxation between electrons and phonons. In this section we apply the model presented in [39] to determine the strength of the electron-phonon coupling. We assume material parameters as given in [39], but apply the nonequilibrium distribution discussed before (see Fig 1) as the starting distribution for the phonons.

In this calculation, only electron-phonon interactions are considered, all other interaction processes are switched off. We calculate the energy transfer rate from the electrons to the phonons according to equation (10) in [39] applying the phonon nonequilibrium distribution as starting distribution while the electrons are Fermi distributed at 10000 Kelvin at the beginning of our calculation. For comparison, the energy transfer rate is also determined assuming equilibrium distributions for both subsystems. In that calculation, at each timestep the distributions of electrons as well as of phonons are set to their corresponding equilibrium distributions of the same internal energy.

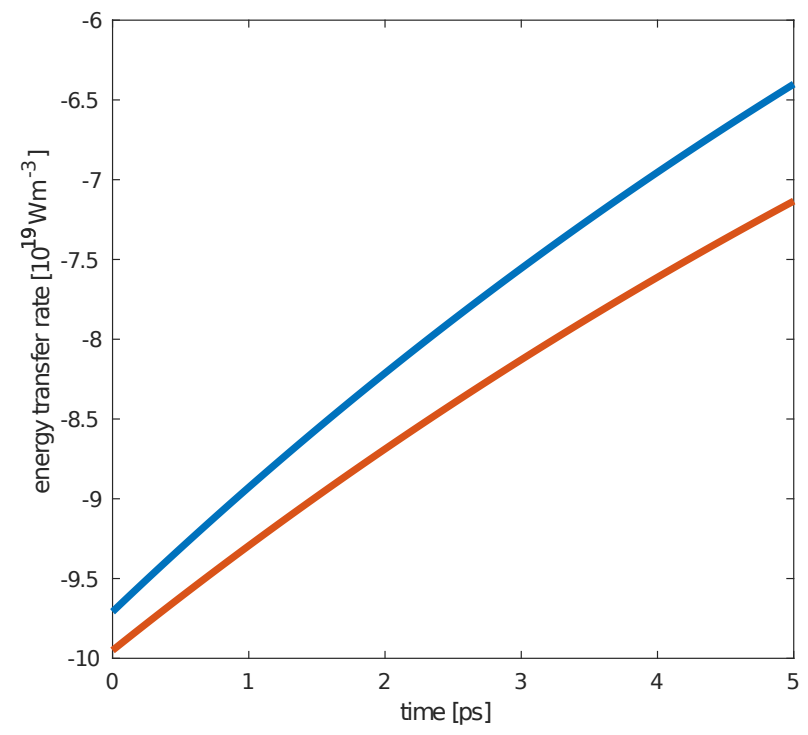

FIG. 6: Rate of the energy transfer from the electrons to the phonons assuming a phonon nonequilibrium (blue) and a Bose distribution at the same internal energy (red).

We observe a considerable difference in the energy transfer rate from the electrons to the phonons depending on the phonon nonequilibrium, as shown in figure 6 . This difference indicates that the phonon nonequilibrium also plays an important role in the evolution of the electron system and the electron-phonon coupling and should therefore not simply be neglected. As a consequence, it is further important to identify a timescale, after which the phonons are thermalized and the assumption of a Bose 
distribution is valid.

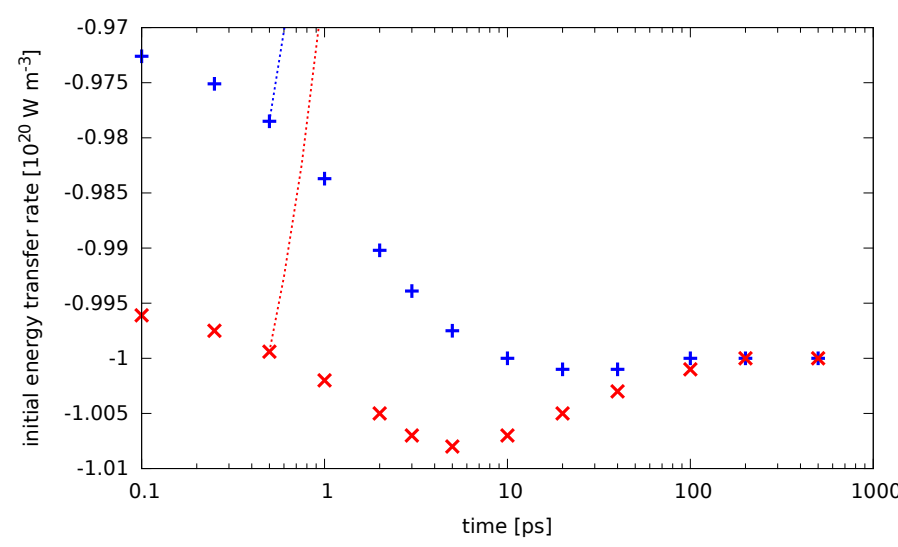

FIG. 7: Rate of the energy transfer from

Fermi-distributed electrons to the nonequilibrium phonons at different timesteps (blue) and

Bose-distributed phonons with the same internal energy (red).

Therefore, the initial energy transfer rates between Fermi distributed electrons and nonequilibrium phonons in different stages of the relaxation process are calculated. We determine the electron-phonon coupling between electrons at 10000 Kelvin and phonons at different nonequilibrium states, calculated according to equation (15) and shown for some delays in Figure 5. With these initial conditions, initial energy transfer rates are calculated. The results are shown in comparison to the coupling to equilibrium phonons of the same respective energy in Figure 7. The blue dots denote the energy transfer rate from the electrons to the phonons in the nonequilibrium distribution at certain timesteps during the relaxation process, the red dots are the energy transfer rates calculated for a Bose distribution of the same respective internal energy. The electrons are always Fermi distributed at 10000 Kelvin. The dashed lines represent the temporal evolution of the energy transfer rate for a certain time, similar to the curves in Figure 6. The energy transfer from the electrons to the phonons is decreasing over time for the phonons in different stages of the thermalization process and the rates for the nonequilibrium dis- tributed phonons are approaching the rates for the Bose distributed phonons, until there is no more observable difference at $200 \mathrm{ps}$. This result indicates, that the assumption of Bose distributed phonons would not make a difference on the electron phonon coupling anymore from this point on. Note that the minimum in the red curve is an artifact due to deviation of the internal energy of the phonons during the calculated relaxation process.

\section{CONCLUSION}

The thermalization of the phonons is studied within the frame of a Boltzmann collision term, which gives a wavenumber-dependent relaxation time. We found these relaxation times to be on a timescale of up to some hundreds of picoseconds. The temporal evolution of a model nonequilibrium phonon distribution after laser irradiation is studied in the framework of a relaxation time approximation. A comparison of the initial energy transfer rates between electrons and phonons in nonequilibrium and thermalized indicates that the thermalization process of the given phonon distribution takes place on a timescale of a few hundred picoseconds. Additionally, we study the energy transfer between Fermi-distributed electrons and nonequilibrium phonons, which is compared to the energy transfer for equilibrium distributions in both subsystems. A comparison of the initial energy transfer rates in both cases indicates that the thermalization process of the given phonon distribution takes place on a timescale of a few hundred picoseconds. In conclusion, we have observed a considerable influence of a thermodynamic nonequilibrium of the phonons on the energy transfer between the electron and phonon systems.

\section{ACKNOWLEDGEMENTS}

The authors thank Nils Brouwer for providing the electron-phonon code. Financial support of the former MBWWK Rheinland-Pfalz, the TU-Nachwuchsring and Deutsche Forschungsgemeinschaft (grant RE 1141/11-1 and RE 1141/15-1) is gratefully acknowledged.
* klett@physik.uni-kl.de

1 T. Brabec and F. Krausz. Intense few-cycle laser fields: Frontiers of nonlinear optics. Rev. Mod. Phys., 72:545-591, 2000.

2 Y. Silberberg. Laser science: Physics at the attosecond frontier. Nature, 414, Nov 2001.

3 D. Bäuerle. Laser Processing and Chemistry. Springer, Berlin, 2011.

4 A. Vogel and V. Venugopalan. Mechanisms of Pulsed Laser Ablation of Biological Tissues. Chem. Rev., 103:577, 2003.
${ }^{5}$ K. Sokolowski-Tinten, J. Bialkowski, M. Boing, A. Cavalleri, and D. von der Linde. Thermal and nonthermal melting of gallium arsenide after femtosecond laser excitation. Phys. Rev. B, 58(18):11805-11808, 1998.

6 A. Cavalleri, C. Toth, C.W. Siders, J.A. Squier, F. Raksi, P. Forget, and C.J. Kieffer. Femtosecond Structural Dynamics in $\mathrm{VO}_{2}$ during an Ultrafast Solid-Solid Phase Transition. Phys. Rev. Lett., 87:237401, 2001.

7 E.S. Zijlstra, A. Kalitsov, T. Zier, and M.E. Garcia. Squeezed Thermal Phonons Precurse Nonthermal Melting of Silicon as a Function of Fluence. Phys. Rev. X, 3:011005, 
2013.

8 B. Rethfeld, A. Kaiser, M. Vicanek, and G. Simon. Ultrafast dynamics of nonequilibrium electrons in metals under femtosecond laser irradiation. Phys. Rev. B, 65:214303, 2002.

9 X. Y. Wang, D. M. Riffe, Y.-S. Lee, and M. C. Downer. Time-resolved electron-temperature measurement in a highly excited gold target using femtosecond thermionic emission. Phys. Rev. B, 50:8016-8019, Sep 1994.

10 C.-K. Sun, F. Vallée, L. H. Acioli, E. P. Ippen, and J. G. Fujimoto. Femtosecond-tunable measurement of electron thermalization in gold. Phys. Rev. B, 50:15337-15348, Nov 1994.

11 A. Kaiser, B. Rethfeld, M. Vicanek, and G. Simon. Microscopic processes in dielectrics under irradiation by subpicosecond laser pulses. Phys. Rev. B, 61:11437, 2000.

12 N. Del Fatti, C. Voisin, M. Achermann, S. Tzortzakis, D. Christofilos, and F. Vallee. Nonequilibrium electron dynamics in noble metals. Phys. Rev. B, 61:16956-16966, 2000.

${ }^{13}$ L. D. Pietanza, G. Colonna, S. Longo, and M. Capitelli. Non-equilibrium electron and phonon dynamics in metals under femtosecond laser pulses. Eur.Phys.J.D, 45:369-389, 2007.

14 Hideo Aoki, Naoto Tsuji, Martin Eckstein, Marcus Kollar, Takashi Oka, and Philipp Werner. Nonequilibrium dynamical mean-field theory and its applications. Rev. Mod. Phys., 86:779-837, Jun 2014.

15 B. Y. Mueller and B. Rethfeld. Relaxation dynamics in laser-excited metals under nonequilibrium conditions. Phys. Rev. B, 87:035139, 2013.

${ }^{16}$ M. Hase, K. Mizoguchi, H. Harima, and S. Nakashima. Dynamics of coherent phonons in bismuth generated by ultrashort laser pulses. Phys. Rev. B, 58(9):5448, 1998.

17 K. Sokolowski-Tinten, C. Blome, J. Blums, A. Cavalleri, C. Dietrich, A. Tarasevitch, I. Uschmann, E. Förster, M. Kammler, M. Horn von Hoegen, and D. von der Linde. Femtosecond x-ray measurement of coherent lattice vibrations near the lindemann stability limit. Nature, 422:287289, 2003.

18 M. Harmand, R. Coffee, M. R. Bionta, M. Chollet, D. French, D. Zhu, D. M. Fritz, H. T. Lemke, N. Medvedev, B. Ziaja, S. Toleikis, and M. Cammarata. Achieving fewfemtosecond time-sorting at hard x-ray free-electron lasers. Nat. Photon., 7:215-218, 2013.

19 Isabel Klett, Tobias Zier, Baerbel Rethfeld, Martin E. Garcia, and Eeuwe S. Zijlstra. Isostructural elemental crystals in the presence of hot carriers. Phys. Rev. B, 91:144303, Apr 2015.

${ }^{20}$ V. Recoules, J. Clerouin, G. Zerah, P. M. Anglade, and S. Mazevet. Phys. Rev. Lett., 96:055503, 2006.

21 E. S. Zijlstra, F. Cheenicode Kabeer, B. Bauerhenne, T. Zier, N. Grigoryan, and M. E. Garcia. Modeling of material properties after ultrashort laser and XUV excitation. Appl. Phys. A, 110:519-528, 2013.

${ }^{22}$ B. Y. Mueller, I. Klett, and B. Rethfeld. Laser-excited metals under non-equilibrium conditions. AIP Conf. Proc., 1464:609, 2012.

23 Rogier H. M. Groeneveld, Rudolf Sprik, and Ad Lagendijk. Effect of a nonthermal electron distribution on the electron-phonon energy relaxation process in noble metals. Phys. Rev. B, 45:5079-5082, Mar 1992.

24 S. I. Anisimov, B. L. Kapeliovich, and T. L. Perel'man. Electron emission from metal surfaces exposed to ultra- short laser pulses. Sov. Phys. JETP, 39(2):375-377, 1974.

25 P. B. Allen. Theory of Thermal Relaxation of Electrons in Metals. Phys. Rev. Lett., 59(13):1460-1463, 1987.

26 Zhibin Lin, Leonid V. Zhigilei, and Vittorio Celli. Electronphonon coupling and electron heat capacity of metals under conditions of strong electron-phonon nonequilibrium. Phys. Rev. B, 77:075133, Feb 2008.

27 J. Hohlfeld, S. S. Wellershoff, J. Güdde, U. Conrad, V. Jähnke, and E. Matthias. Electron and lattice dynamics following optical excitation of metals. Chem.Phys., 251:237-258, 2000.

${ }^{28}$ Lutz Waldecker, Roman Bertoni, Ralph Ernstorfer, and Jan Vorberger. Electron-phonon coupling and energy flow in a simple metal beyond the two-temperature approximation. Phys. Rev. X, 6:021003, Apr 2016.

29 E. Carpene. Ultrafast laser irradiation of metals: Beyond the two-temperature model. Phys. Rev. B, 74:024301, Jul 2006.

${ }^{30}$ V. V. Kabanov and A. S. Alexandrov. Electron relaxation in metals: Theory and exact analytical solutions. Phys. Rev. B, 78:174514, Nov 2008.

31 V. V. Baranov and V. V. Kabanov. Theory of electronic relaxation in a metal excited by an ultrashort optical pump. Phys. Rev. B, 89:125102, Mar 2014.

32 N. S. Shcheblanov and T. E. Itina. Femtosecond laser interactions with dielectric materials: insights of a detailed modeling of electronic excitation and relaxation processes. Appl. Phys. A, 110:579-583, 2013.

33 N. Medvedev, U. Zastrau, E. Förster, D. O. Gericke, and B. Rethfeld. Short-time electron dynamics in aluminum excited by femtosecond extreme ultraviolet radiation. Phys. Rev. Lett., 107:165003, Oct 2011.

34 Shota Ono. Nonequilibrium phonon dynamics beyond the quasiequilibrium approach. Phys. Rev. B, 96:024301, Jul 2017.

35 J. M. Ziman. Electrons and Phonons. Oxford University Press, 1962.

36 J. M. Manceau, P. A. Loukakos, and S. Tzortzakis. Direct acoustic phonon excitation by intense an ultrashort terahertz pulses. Appl. Phys. Lett., 97:251904, 2010.

37 F. H. Stillinger and T. A. Weber. Computer simulation of local order in condensed phases of silicon. Phys. Rev. B, 31(8):5262, 1985.

38 A. Giri and P. E. Hopkins. Transient thermal and nonthermal electron and phonon relaxation after short-pulsed laser heating of metals. J. Appl. Phys., 118:215101, 2015.

39 Nils Brouwer and Baerbel Rethfeld. Transient electron excitation and nonthermal electron-phonon coupling in dielectrics irradiated by ultrashort laser pulses. Phys. Rev. $B$, 95:245139, Jun 2017. 